\title{
A refined hemispheric model of normal human aortic valve and root geometry
}

\author{
J. Scott Rankin, MD, ${ }^{a}$ M. Crockett Bone, BS, ${ }^{a}$ Peter M. Fries, MD, ${ }^{b}$ Diana Aicher, MD, ${ }^{b}$ \\ Hans-Joachim Schäfers, MD, ${ }^{\mathrm{b}}$ and Philip S. Crooke, $\mathrm{PhD}^{\mathrm{a}}$
}

Background: Better understanding of aortic root geometry could improve diagnosis and reconstruction of pathologic aortic valves. In this study, a previous model of hemispheric aortic valve leaflets nested within a cylindrical aorta was refined in humans with normal aortic valves.

\begin{abstract}
Methods: Using 1-mm axial slices, high-resolution computed tomographic angiograms from 10 normal aortic roots were used to generate high-density X-, Y-, and Z-coordinates of valve structures using Mathematica software. Three-dimensional least squares regression analyses of leaflet and sinus coordinates were employed to compare multiple geometric models of aortic valve and root geometry. Shapes and dimensions of all root structures were evaluated and compared.
\end{abstract}

Results: Aortic valve geometry was roughly hemispherical, but the valve base was elliptical (minor-major diameter ratio $=.66$ ). Dimensional fits of the leaflet-sinus complexes also were better using ellipsoidal geometry, with taller leaflets than predicted by hemispheres. The commissure between the left and noncoronary cusps was located uniformly at the posterior junction of the base minor diameter and circumference, with the center of the right coronary cusp opposite. The subcommissural post areas flared outward by $5^{\circ}$ to $10^{\circ}$, and the volume of the right coronary leaflet-sinus complex was $12.4 \%$ and $10.7 \%$ larger than the noncoronary cusps and left cusps, respectively.

Conclusions: The normal human aortic valve is an elliptical structure, and ellipsoidal refinements improve representation of leaflet geometry. The left and noncoronary cusps commissure is located posteriorly; the right coronary cusp is located anteriorly. This model could be useful in quantifying pathologic geometry and in engineering devices for aortic valve reconstruction. (J Thorac Cardiovasc Surg 2013;146:103-8)

\section{Supplemental material is available online.}

Reconstruction of aortic valves is being performed increasingly, especially for aortic insufficiency. ${ }^{1-4}$ Stability and reproducibility of repair are improving, and data suggest that outcomes are enhanced over valve replacement. ${ }^{5}$ Further progress may be limited by lack of a precise understanding of aortic valve geometry. Most existing analyses were obtained either at autopsy or in animals. ${ }^{6-14}$ With recent improvements in clinical imaging methods, it is

\footnotetext{
From Centennial Medical Center, ${ }^{a}$ Vanderbilt University, Nashville, Tenn; and University of Saarland, ${ }^{\mathrm{b}}$ Homburg/Saar, Germany.

This study was funded by BioStable Science and Engineering Inc, Austin, Tex. Disclosures: Dr Rankin is a consultant and chief medical officer for BioStable Science and Engineering, Inc. Dr Crooke is a consultant. The other authors have nothing to disclose with regard to commercial support. All authors had complete scientific freedom in experimental design, data acquisition, data analysis, and publication. Presented as a poster at the Society of Heart Valve Disease meeting, Barcelona, Spain, Monday, June 27, 2011.

Received for publication March 28, 2012; revisions received May 12, 2012; accepted for publication June 15, 2012; available ahead of print Aug 2, 2012.

Address for reprints: J. Scott Rankin, MD, 320 Lynnwood Blvd, Nashville, TN 37205 (E-mail: jsrankinmd@cs.com).

$0022-5223 / \$ 36.00$

Copyright (c) 2013 by The American Association for Thoracic Surgery http://dx.doi.org/10.1016/j.jtcvs.2012.06.043
}

possible to assess aortic valve geometry in normal awake humans. Thus, this study was designed to evaluate and refine a previously described hemispheric model of aortic valve geometry ${ }^{15}$ in awake patients with normal aortic valves.

\section{METHODS}

This study was approved by Western Institutional Review Board. Ten patients undergoing screening computed tomographic (CT) angiography for coronary artery disease were selected randomly for study. Patients were 7 men and 3 women ranging in weight from 135 to $225 \mathrm{lb}$. No patient was defined clinically as having aortic valve disease. Thoracic CT angiographic images were obtained with a high-resolution Siemens Definition Dual 64 slice CT scanner (Siemens AG, Munich, Germany) gated to end-diastole. Radiographic data were acquired with a coronary imaging protocol approximately 40 seconds after peripheral intravenous injection of contrast solution. Transverse slice orientation of the CT images across the central axis of the valve and ascending aorta was accomplished by having the base of all 3 valve leaflets first appear in the same CT slice. Axial end-diastolic CT slices were obtained with 1-mm thickness from the outflow tract of the left ventricle to beyond the sinotubular junction (Figure E1). The group of Dicom images produced by the CT scanner were de-identified and converted to an audio video interleave file using a Diacom (digital imaging and communications in medicine) viewer. The audio video interleave (.avi) file for each valve then was imported into Adobe Premiere (Adobe Systems, Mountain View, Calif), and individual bitmap files were generated by the software each time the image changed (1-mm intervals). The bitmap files then were imported into Mathematica (Wolfram Research, Inc, Champaign, Ill) for analysis. 


$$
\begin{aligned}
& \text { Abbreviations and Acronyms } \\
& \text { CT }=\text { computed tomography } \\
& \text { LC }=\text { left coronary } \\
& \text { NC }=\text { noncoronary } \\
& \text { RC }=\text { right coronary }
\end{aligned}
$$

All quantitative data were generated in a custom computer program for Mathematica. Bitmap images from each 1-mm CT slice were selected throughout the long axis of the valve, and all valve structures for each 1-mm slice were defined by discrete points. A 20 -mm calibration view also was digitized for conversion of distances between data points to quantitative dimensions. As shown in Figure 1, points were placed as densely as possible to outline each structure, and aortic or subcommissural points were identified by red dots. Right, left, and noncoronary sinus structures were defined by green, yellow, and orange Xs within circles, respectively. Right, left, and noncoronary leaflets were traced with green, yellow, and orange dots within circles, respectively (Figure 1). This arrangement produced calibrated quantitative $\mathrm{X}-\mathrm{Y}$ coordinates of valve structures for each 1-mm CT slice, and the 1-mm CT slice value produced the Z-coordinate. Thus, calibrated high density $\mathrm{X}, \mathrm{Y}$, and Z 3-dimensional coordinates in space could be generated for individual structures within the entire valve.

Three-dimensional least squares regression analyses were applied to multiple structures to reconstruct the valve. First, the cross section of the left ventricular outflow tract/valve base was modeled with circular and elliptical equations. Similarly, each leaflet sinus complex was assessed with a hemispheric model, ${ }^{15}$ and then with general ellipsoid equations. Goodness of fit for the 2 models was compared by computing the sum of residual squares. The results of the least squares fitting were quadratic equations:

Hemispheric model

$$
a_{i}\left(x^{2}+y^{2}+z^{2}\right)+b_{i} x+c_{i} y+d_{i} z+e_{i}=0, \quad i=1,2,3
$$

Ellipsoid model

$$
a_{i} x^{2}+b_{i} y^{2}+c_{i} z^{2}+2 f_{i} y z+2 g_{i} x z+2 h_{i} x y+2 p_{i} x+2 q_{i} y+2 r_{i} z+d_{i}=0,
$$

$$
i=1,2,3
$$

where the coefficients of $x, y$, and $z$ terms come from the regression analysis. An equation of these 2 types existed for each leaflet, and the parameters in this equation, $a_{i}, b_{i}, c_{i}, f_{i}, g_{i}, h_{i}, p_{i}, q_{i}, r_{i}, d_{i}$, were used to calculate the various properties of the ellipsoid; for example, the 3 axes, center, volume, surface area. Thus, the surface area was found by computing numerically an elliptical integral.

The coaptation planes were obtained mathematically by finding the intersection of the of the leaflet equations. That is, one solves

$$
\begin{gathered}
a_{i} x^{2}+b_{i} y^{2}+c_{i} z^{2}+2 f_{i} y z+2 g_{i} x z+2 h_{i} x y+2 p_{i} x+2 q_{i} y+2 r_{i} z+d_{i} \\
=a_{j} x^{2}+b_{j} y^{2}+c_{j} z^{2}+2 f_{j} y z+2 g_{j} x z+2 h_{j} x y+2 p_{j} x+2 q_{j} y \\
+2 r_{j} z+d_{j, i} \neq j,
\end{gathered}
$$

for fixed values of $z$. For each value of $z$, this gives 2 points, $\left\{\left(x_{1}, y_{1}\right)\left(x_{2}, y_{2}\right)\right\}$, from which the lines in the intersection plane joining the 2 points were constructed. These lines were used to approximate the area of coaptation. The calculation of the least squares equations for

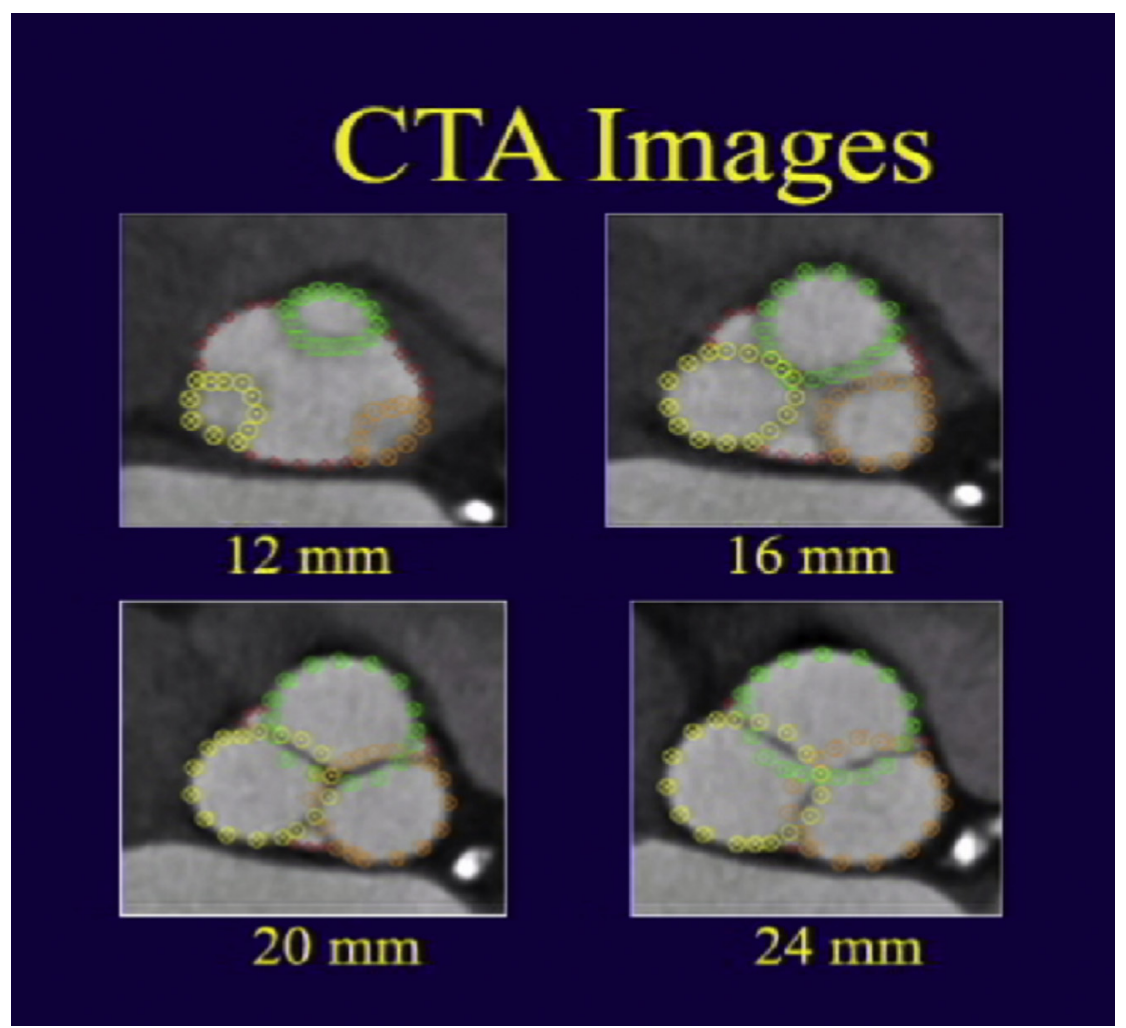

FIGURE 1. Representative transverse computed tomographic angiographic (CTA) slices of an aortic valve at 4 mm intervals along the axis of the valve. Using Mathematica software (Wolfram Research, Inc, Champaign, Ill), individual colored points were placed in high-density to define specific aortic root structures for regression analyses. 
TABLE 1. Average valve data derived from transverse axial computed tomographic angiographic slices in 10 patients

\begin{tabular}{|c|c|c|c|c|c|c|c|c|}
\hline \multirow[b]{2}{*}{ Structure } & \multirow{2}{*}{$\begin{array}{c}\text { Base } \\
\text { geometry* }\end{array}$} & \multicolumn{3}{|c|}{ Leaflet region } & \multicolumn{3}{|c|}{ Commissural region } & \multirow{2}{*}{$\begin{array}{c}P \\
\text { value }\end{array}$} \\
\hline & & NC & $\mathbf{R C}$ & $\mathbf{L C}$ & L-R & N-R & N-L & \\
\hline Circumference & $73.7 \pm 11.1$ & - & - & - & - & - & - & - \\
\hline Circumference segment & - & $24.0 \pm 3.6$ & $24.0 \pm 3.7$ & $25.8 \pm 5.1$ & \multicolumn{3}{|c|}{ (Kruskal-Wallace test) } & .003 \\
\hline Height & - & $24.7 \pm 2.2$ & $24.6 \pm 1.8$ & $25.1 \pm 3.0$ & \multicolumn{3}{|c|}{ (K-Sample $t$ test) } & .85 \\
\hline Major diameter & $28.2 \pm 5.4$ & $18.7 \pm 2.1$ & $19.4 \pm 2.2$ & $20.6 \pm 2.1$ & \multicolumn{3}{|c|}{ (K-Sample $t$ test) } & .14 \\
\hline Minor diameter & $18.2 \pm 2.2$ & $16.2 \pm 2.1$ & $15.5 \pm 1.5$ & $16.3 \pm 2.1$ & \multicolumn{3}{|c|}{ (Kruskal-Wallace test) } & .27 \\
\hline $\begin{array}{l}\text { Least square error } \\
\text { (sphere) }\end{array}$ & - & $15.3 \pm 2.8$ & $18.7 \pm 6.7$ & $12.8 \pm 1.3$ & \multicolumn{3}{|c|}{ (Kruskal-Wallace test) } & .003 \\
\hline $\begin{array}{l}\text { Least square error } \\
\text { (ellipsoid) }\end{array}$ & - & $8.3 \pm 1.6$ & $8.2 \pm 1.6$ & $7.7 \pm 1.5$ & \multicolumn{3}{|c|}{ (K-Sample $t$ test) } & .62 \\
\hline Leaflet surface area & - & $616 \pm 87$ & $670 \pm 119$ & $619 \pm 110$ & \multicolumn{3}{|c|}{ (K-Sample $t$ Test) } & .42 \\
\hline Leaflet-sinus volume & - & $1960 \pm 425$ & $2238 \pm 601$ & $1998 \pm 545$ & \multicolumn{3}{|c|}{ (K-Sample $t$ test) } & .42 \\
\hline Minor/major ratio & 0.66 & $0.87 \pm 0.04$ & $0.80 \pm 0.11$ & $0.79 \pm 0.12$ & \multicolumn{3}{|c|}{ (Kruskal-Wallace test) } & .01 \\
\hline$\alpha$ & - & $.32 \pm .14$ & $.09 \pm .21$ & $.24 \pm .07$ & \multicolumn{3}{|c|}{ (Kruskal-Wallace test) } & .003 \\
\hline Commissural angles $\left(^{\circ}\right)$ & & & & & $3.9 \pm 2.7$ & $5.0 \pm 2.8$ & $\begin{array}{c}7.6 \pm 4.8 \\
(\mathrm{~K}-\text {-Sample } t \text { test })\end{array}$ & .06 \\
\hline Leaflet coaptation area & & & & & $51.0 \pm 9.3$ & $57.9 \pm 19.9$ & $\begin{array}{c}29.3 \pm 15.1 \\
\text { (Kruskal-Wallace test) }\end{array}$ & .002 \\
\hline
\end{tabular}

Data presented as mean \pm standard deviation; dimensions are average values in 10 normal humans in millimeters, areas are in millimeters squared, and volumes are in millimeters cubed. $N C$, Noncoronary; $R C$, right coronary; $L C$, left coronary; $L-R$, left-right coronary; $N$ - $R$, non-right coronary; $N$ - $L$, non-left coronary. *Average diameters refer to minor and major base diameters along with their ratio. †Each statistical test was selected by Mathematica software (Wolfram Research Inc, Champaign, Ill) as the most appropriate for the specific data.



FIGURE 2. Aortic valve geometry. A, Representative computed tomographic angiography of a normal valve with coordinates placed. B, Average dimensions of valves analyzed in this study; green ellipse is valve base, black points are commissures, purple circle is the right coronary leaflet-sinus complex, and blue dots are the centrums of the various leaflet sinus complexes. C, Green ellipse is the elliptical valve base; blue vertical lines are the centrums of the leaflet-sinus complexes; red is the leaflet aortic junction, or the valve annulus. D, Model of a reconstructed valve; orange structure represents the right coronary leaflet-sinus complex and the brown is the elliptical valve base. 


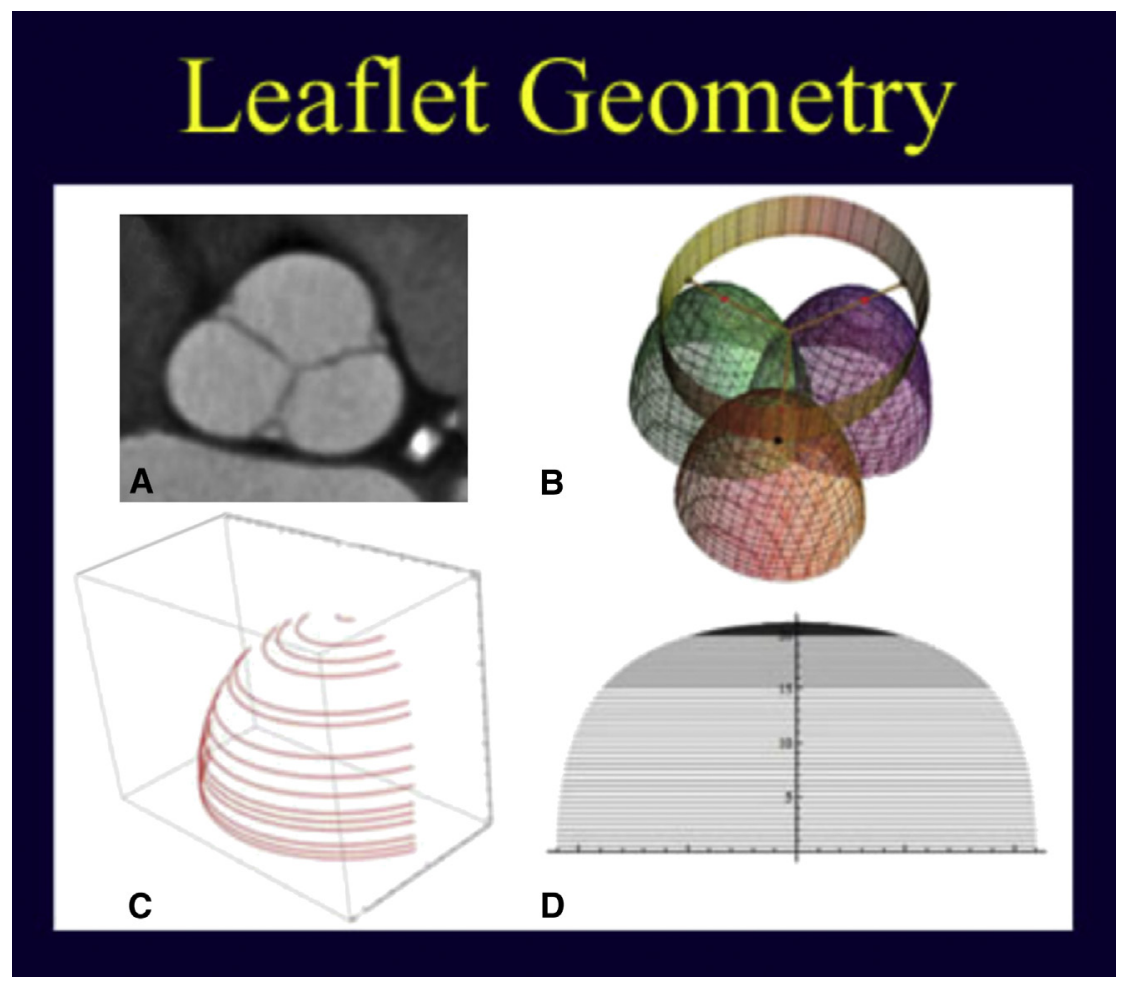

FIGURE 3. Leaflet geometry. A, computed tomographic angiographic frame from which the geometry is derived. B, View of the reconstructed valve from below showing leaflet geometry. C, An infinite number of transverse lines were placed on the leaflet. D, leaflet geometry after the lines are placed on a flat surface.

each leaflet used only points on the leaflet that were not in coaptation. Given the lateral leaflet insertions on the aorta and the large areas of leaflets not in coaptation, ample data were available to predict the ellipsoid geometry of the entire leaflet. Thus, leaflet extensions beyond the planes of coaptation shown in all Figures were mathematical constructions of predicted noncoapted leaflet geometry.

In the ellipsoid analysis, the major and minor diameters of both the valve base and each leaflet sinus complex were calculated. Threedimensional volumes of the 3 leaflet-sinus complexes were calculated as well as leaflet vertical height. Fractional migration of each leaflet-sinus complex centrum from the base circumference toward the center of the valve was described by the term $\alpha$. Average valve and leaflet dimensions for the 10 valves were generated \pm 1 standard deviation, including leaflet areas, coaptation areas, and commissural angle deviations relative to the long axis of the valve. Differences were compared using KruskalWallace or K-Sample $t$ tests.

\section{RESULTS}

Images of 4 transverse axial slices of a representative valve are shown in Figure 1. Images were clear using the high-resolution scanner, and structures were easily definable. Again, the coapted leaflet positions were entered initially, and then the predicted (uncoapted) leaflet geometry was derived from the ellipsoidal regression data. The valve base was very elliptical (Figure E2), and valve leaflet-sinus complexes, reconstructed with the least squares regression analyses, were better represented with ellipsoid least squares regressions than with hemispheric models, achieving half the sum of squares values generally (Figure E2) (see Table 1). For all 3 leaflets, vertical leaflet height was greater than represented by hemispheres. Leaflet-sinus cross sections were not quite circular either (Table 1), and cross-sectional minor/major diameter ratio averaged 0.79 to 0.87 for the 3 leaflets $(P<.01)$, with the larger diameter being the intercommissural distance. Geometric shapes, dimensions, areas, and volumes among the 3 leaflets were quite similar, except the right coronary (RC) leaflet averaged $\sim 10 \%$ larger than the other 2 (see Table 1 and Figure 2, A), although this difference did not reach statistical significance $(P=.42)$.

The transverse geometry of the base of the valve at first appearance of the leaflets was uniformly elliptical, rather than circular, with an average minor to major diameter ratio of 0.66 (see Table 1 and Figure 2,B). The average elliptical circumference was $73.7 \pm 11.1 \mathrm{~mm}$, which is equivalent to a circular diameter of $23.5 \mathrm{~mm}$. The commissure between the left coronary (LC) and noncoronary (NC) leaflets was always located at the posterior minor diameter-circumference junction, with the center of the RC leaflet opposite (Figure 2, $B$ and $C$ ). The centrum of each leaflet-sinus complex was migrated toward the center of the valve for all leaflets, but to a lesser extent for the RC leaflet (Figure 2, $B$ and $C)$. Average $\alpha$ values were: $\mathrm{NC}=.32, \mathrm{RC}=.09$, and $\mathrm{LC}=.24(P<.01 \mathrm{RC}$ vs others $)$. The subcommissural 


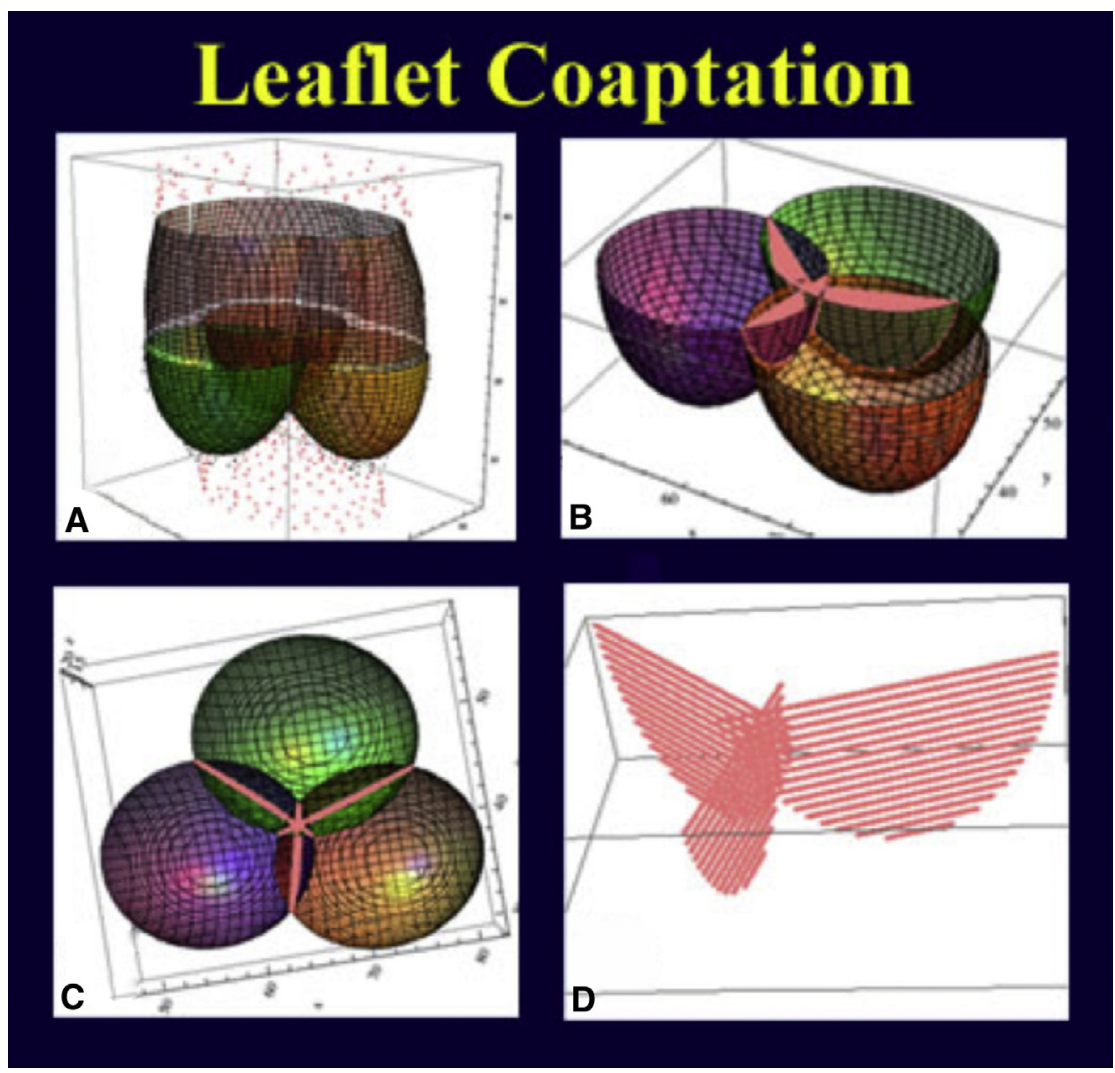

FIGURE 4. Leaflet coaptation. A, computed tomographic angiographic frame from which the geometry is derived. B, View of the reconstructed valve from below showing leaflet geometry. C, A different perspective of the intersecting leaflets. D, A set of transverse lines was calculated from the curve that is formed from the intersection of 2 leaflets. In all of the Figures, the parts of the leaflets beyond the plane of coaptation were predicted by the regression analyses and are purely mathematical.

regions of the valve flared outward from the long axis of the valve by a consistent angle of $5^{\circ}$ to $8^{\circ}$ degrees from vertical (Table 1); thus, the ellipticality of the valve extended up the commissures to the top of the valve. The distance on the circumference between commissural posts was statistically larger for the left cusp, but from a practical viewpoint, the 3 intercommissural distances proved to be approximately equivalent in normal valves (Table 1). Presence of the 3 circular leaflet sinus complexes made the base ellipticality difficult to perceive (Figure 2, D), but it was present in every patient. In Figure 3, leaflet shape was evaluated by constructing several transverse leaflet lines and then laying the lines down flat. Leaflet shape, evaluated with this approach, was broader and more rectangular than shown previously. Finally, the 2 coaptation surfaces involving the RC leaflet were uniformly larger than the posterior NC-LC coaptation surface (see Figure 4 and Table 1) $(P<.01)$.

\section{DISCUSSION}

Since Leonardo DaVinci's anatomic drawings, the trileaflet structure of semilunar valves has been fairly well understood. Numerous autopsy and animal studies have contributed further to this knowledge. With the advent of high-resolution CT angiography, evaluation and analyses of aortic valve geometry in a more physiologic setting became possible. The 3-dimensional coordinates of all valve structures in awake patients with normal valves were imported into Mathematica and subjected to precise numeric evaluation. The findings of this study were somewhat surprising. Having examined human aortic valves angiographically and with direct surgical vision for many decades, the basic ellipticality of the valve had not been appreciated. Perhaps the nesting of three circular leaflet-sinus complexes obscured the elliptical base geometry, but certainly, the elliptical nature of the valve was a surprise. Furthermore, the ellipticality extended vertically to the top of the commissures, and along with the inward leaflet migration (the $\alpha$ factor), the elliptical base geometry seemed to force the leaflets together and facilitate normal leaflet coaptation. Perhaps loss of ellipticality could be important in pathologic root dilatation and the creation of valvular insufficiency, and more studies, especially under pathologic conditions, seem indicated.

Other surprises were evident. The specific anatomic arrangement of the $\mathrm{LC}-\mathrm{NC}$ commissure being located on 
the posterior minor diameter was unanticipated, along with the broader-appearing RC leaflet opposite. It was thought that significant asymmetry could be common, but in fact, the commissures in the normal valve were positioned approximately equidistant around the circumference, and leaflet shapes, areas, and volumes also were similar (with the RC being only $10 \%$ larger, on average). Finally, the outward flare of the commissures was unanticipated, and could be important for the suspension of leaflets for adequate coaptation. Overall, a new picture of aortic valve anatomy emerged, one that was quite specific for the definition of "normality." Certainly, variability is bound to exist, but within the 10 normal valves examined in this study, valve geometry was very consistent.

These findings could be important in the design of future devices for aortic valve repair. Any geometric aortic annuloplasty device ${ }^{16-18}$ would seem to require an elliptical shape. In fact, restoration of valve ellipticality could be important for adequate trileaflet aortic valve coaptation, as it is in the mitral valve. ${ }^{19}$ Restoration of outwardly flaring commissures could be important, as well as equidistant spacing of commissures on the base circumference. Given the fairly symmetrical leaflet size and spacing observed in normal valves, perhaps much of the asymmetry noted in diseased valves could be due to pathologic/asymmetric root or sinus dilatation. It is possible that restoration of precise annular geometry and symmetry, together with well-established leaflet repair procedures, ${ }^{1-4}$ could be important in correcting pathologic aortic valves back to optimal coaptation. In the final analysis, more precise measurements will need to be made in pathologic states, and the various geometric hypotheses tested in diseased valves.

\section{CONCLUSIONS}

Geometric analyses of normal human aortic valves identified a very specific anatomy that included ellipticality, high degrees of symmetry, and outwardly flaring commissures. Consideration of these factors could improve the development of techniques and devices for autologous aortic valve repair.

\section{References}

1. Shäfers H-J, Aicher D, Langer F. Correction of leaflet prolapse in valve preserving aortic replacement: pushing the limits? Ann Thorac Surg. 2002; 74:1762-74.

2. Schäfers H-J. Reconstruction of the bicuspid aortic valve. Op Tech Thorac Cardiovasc Surg. 2007;12:2-13.

3. Aicher D, Langer F, Adam O, et al. Cusp repair in aortic valve reconstruction: does the technique affect stability? J Thorac Cardiovasc Surg. 2007;134:1533-9.

4. Rankin JS, Gaca JG. Techniques of aortic valve repair. Innovations. 2011;6: 348-54.

5. Aicher D, Fries R, Rodionycheva S, et al. Aortic valve repair leads to a low incidence of valve-related complications. Eur J Cardiothorac Surg. 2010;37:127-32.

6. Thurbrikar M, Piepgrass WC, Shaner TW, et al. The design of the normal aortic valve. Am J Physiol. 1981;241:H795-801

7. Kunzelman KS, Grande J, David TE, et al. Aortic root and valve relationships. J Thorac Cardiovasc Surg. 1994;107:162-70.

8. Sutton JP, Ho SY, Anderson RH. The forgotten interleaflet triangles: a review of the surgical anatomy of the aortic valve. Ann Thorac Surg. 1995;59:419-27.

9. Grande KJ, Cochran RP, Reinhall PG, et al. Stress variations in the human aortic root and valve: the role of anatomic asymmetry. Ann Biomed Engineering. 1998; 26:534-45.

10. Beck A, Thubrikar MJ, Robicsek F. Stress analysis of the aortic valve with and without the sinuses of Valsalva. J Heart Valve Dis. 2001;10:1-11.

11. Nicosia MA, Cochran RP, Einstein DR, et al. A coupled fluid-structure finite element model of the aortic valve and root. J Heart Valve Dis. 2003;12:781-9.

12. Labrosse MR, Beller CJ, Robicsek F, et al. Geometric modeling of functional trileaflet aortic valves: development and clinical applications. J Biomech. 2006;39: 2665-72.

13. Vollebergh FE, Becker AE. Minor congenital variations of cusp size in tricuspid aortic valves. Possible link with isolated aortic stenosis. Br Heart J. 1977;39: 1006-11.

14. Anderson RH. Clinical anatomy of the aortic root. Heart. 2000;84:670-3.

15. Rankin JS, Dalley AF II, Crooke PS, et al. A "hemispherical" model of aortic valve geometry. J Heart Valve Dis. 2008;17:179-86.

16. Rankin JS. An intra-annular "hemispherical" annuloplasty frame for aortic valve repair. J Heart Valve Dis. 2010;19:97-103.

17. Rankin JS, Beavan LA, Cohn WE. Technique for aortic valve annuloplasty using an intra-annular "hemispherical" frame. J Thorac Cardiovasc Surg. 2011;142: 933-6.

18. Rankin JS, Conger JL, Tuzun E, et al. In vivo testing of an intra-annular aortic valve annuloplasty ring in a chronic calf model. Eur J Cardiothorac Surg. 2012;42:149-54.

19. Rankin JS, Gaca JG, Daneshmand MA, et al. Increasing mitral valve repair rates with non-resectional techniques. Innovations. 2011;6:209-20. 




FIGURE E1. Orientation of transverse $1 \mathrm{~mm}$ computed tomographic angiographic $(C T A)$ slices out the longitudinal axis of the aortic valve and root.

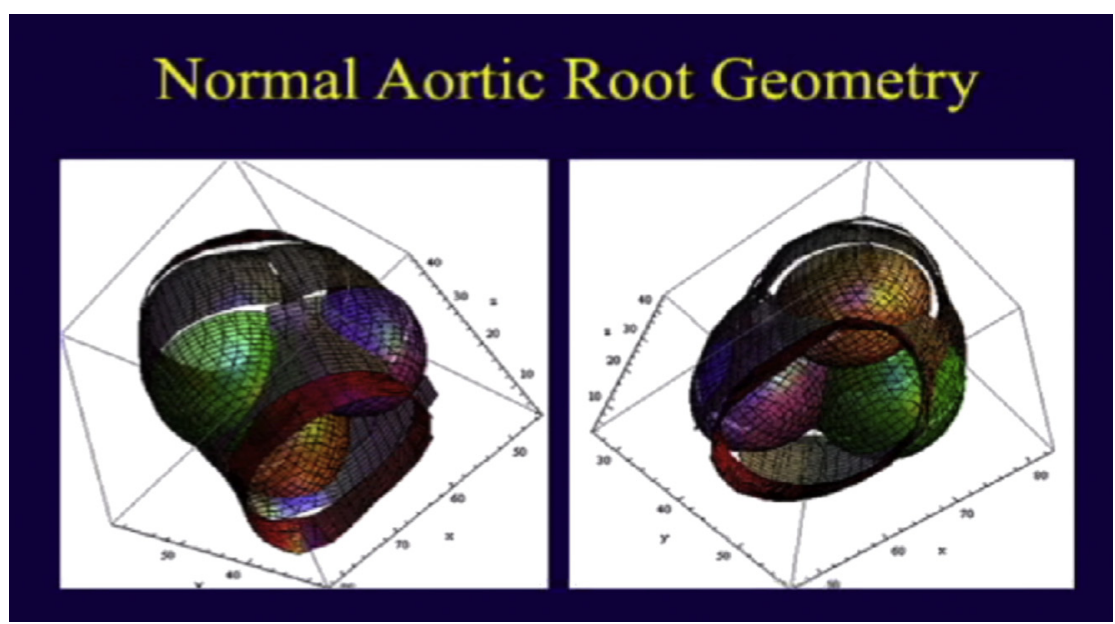

FIGURE E2. A representative valve reconstructed with ellipsoid models. The green leaflet-sinus complex is the right cororay, the yellow is the noncoronary, and the purple is the left coronary. The left ventricular outflow tract is shown in red. 